\title{
The management of thoracic injuries
}

\author{
D. B. Clarke \\ F.R.C.S. \\ Lecturer in Surgery, \\ Queen Elizabeth Hospital, Birmingham
}

WITH THE roads daily becoming choked with fast cars, Casualty Departments throughout the country are increasingly familiar with major chest injuries. The crushing forces applied to the chest as the driver is thrown against the steering column, and the stresses imposed upon mediastinal structures by rapid deceleration in head-on collision often produce injuries so severe that death is instantaneous. In those fortunate enough to reach hospital, resuscitative measures must be applied immediately and often by doctors untrained in thoracic surgery. London (1963) found ninetythree chest injuries in a series of 551 casualties admitted to the Birmingham Accident Hospital, and of these, sixty-eight had other important lesions. The principles underlying the management of thoracic trauma are straightforward and their application involves only a few easily acquired techniques. Abrams (1961a) succinctly defined the causes of death from chest injury as 'the lethal triad, bleeding, drowning and suffocation', and it is under these headings that the treatment of thoracic injuries will be considered.

\section{Bleeding}

Blood loss acquires an additional significance when respiration is impaired by damage to the lungs and chest wall. The loss of a pint of blood may be critical in a patient already hypoxic because of crippled ventilation, for not only is oxygen transport impeded, but the diminution in cardiac output associated with haemorrhage may lead to systemic capillary vasoconstriction and poor tissue perfusion. This gives rise to a metabolic acidosis in addition to the respiratory acidosis associated with lung trauma (Fig. 1). Blood replacement must be swift and adequate. Two or more litres of blood can easily be retained within the pleural cavity, and such bleeding may be readily recognized, both clinically and radiographically. Less obvious is haemorrhage into the chest wall. A litre of blood can be lost from torn intercostal muscles and accommodated within the planes between the chest wall muscles without producing much swelling. There is misplaced re- luctance to transfuse blood rapidly in the presence of suspected pulmonary or cardiac injury. The heart is more likely to fail if its own coronary blood flow is in jeopardy because of hypovolaemia. Rapid blood replacement is vital. Measurement of the central venous pressure by means of a polythene catheter inserted into the external jugular or any large vein gives a good guide to the adequacy of transfusion. The association of a high venous pressure with a low systemic arterial pressure should alert the surgeon to the presence of cardiac tamponade or heart failure from cardiac contusion.

Blood contained within the pleural cavity will compress the lung and further threaten the patient's oxygenation. Re-expansion of the lung must be attained as quickly as possible. Aspiration by a needle and syringe is slow, and may need

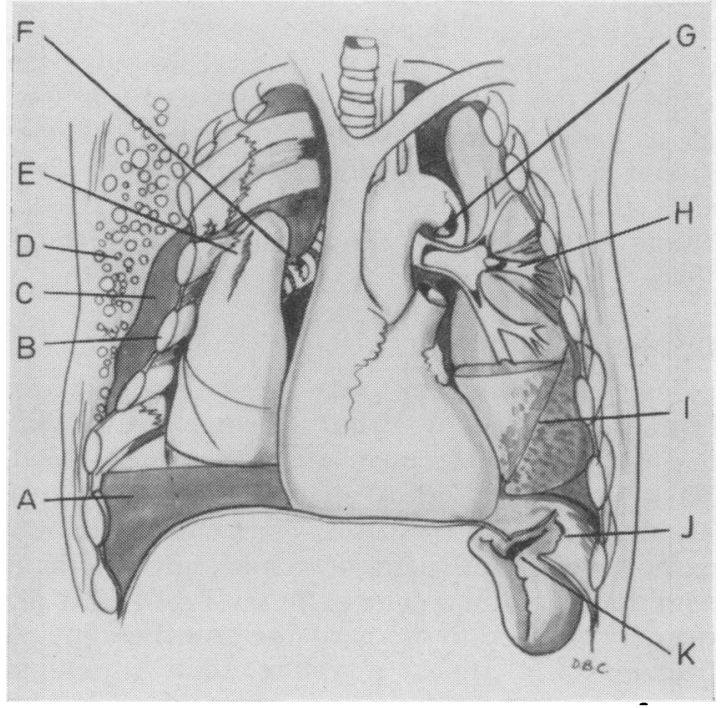

Fig. 1. Composite diagram of the results of chest injury. A, haemothorax; B, stove-in chest; C, chest wall haematoma; D, surgical emphysema; $E$, laceration of the lung with pneumothorax; $F$, rupture of the bronchus; $\mathrm{G}$, rupture of the aorta; $\mathbf{H}$, obstruction of a bronchus by inhaled blood with distal atelectasis; I, 'crushed lung'; $\mathrm{J}$, ruptured diaphragm; $\mathrm{K}$, ruptured abdominal viscus. 
to be repeated if bleeding continues. By far the most satisfactory method of dealing with intrapleural haemorrhage is to insert an intercostal catheter, connected to an underwater seal drainage bottle. Not only will blood be evacuated quickly, but continuous drainage enables the surgeon to know whether bleeding is continuous and, if so, at what rate. Further, the intercostal tube is ideal for removing any air which may be escaping from a laceration of the lung (Beall, Crawford \& DeBakey, 1966).

The chest wall is infiltrated with $20 \mathrm{ml}$ of local anaesthetic in the posterior axillary line level with the nipple. A wide-bore trocar and cannula is inserted through a short skin incision and thrust through an intercostal space into the chest.

A whistle-tip catheter is passed through the cannula, which is then withdrawn and the catheter is anchored with an encircling suture and strapping. If a haemopneumothorax is present a second catheter is inserted through the second intercostal space anteriorly in order to remove the air. Suction is applied to the underwater seal bottle. Blood drainage from the chest is recorded every $15 \mathrm{~min}$. If bleeding of the order of $200-300 \mathrm{ml} / \mathrm{hr}$ continues without diminishing for 3 or $4 \mathrm{hr}$, or if haemorrhage is so brisk that there is difficulty in keeping pace with it by transfusion, it is wisest to resort to thoracotomy. Such bleeding is usually systemic in origin, coming from either the internal mammary or intercostal arteries. Bleeding from the lung itself is seldom severe or persistent. Where removal of blood from the pleura has not been achieved, the lung will be compressed by a shell of clot which may become infected. This clot should be removed by thoracotomy a few days after the injury when the patient's general condition is satisfactory (Price-Thomas \& Cleland, 1945).

\section{Drowning}

The injured patient may drown in his own bronchial secretions, from bleeding within the bronchial tree or from inhaled vomitus or blood. Blood will collect in the pharynx when a fracture of the posterior fossa is compound into the nasopharynx. The signs of coarse rales, perhaps with atelectasis of a lobe or lung, should alert the casualty surgeon to the need for bronchoscopy. A fracture of a rib in a young adult is seldom dangerous. In an elderly bronchitic, the pain from such a fracture will impair coughing and limit respiratory excursion. Sputum retention will be followed by carbon dioxide retention and, eventually, respiratory failure. It is a cardinal error to immobilize the chest with adhesive strapping. Small doses of analgesics will usually relieve the pain enough for the patient to co-operate with the physiotherapist, or if this fails, the fracture site can be infiltrated with local anaesthetic or the appropriate intercostal nerves can be blocked. Epidural anaesthesia is popular in America for the relief of pain.

In the ward, drowning can occur if the patient is left flat on his back, unable to cough because of pain. If he is unconscious from an associated head injury, the danger is greater still. More bronchi can drain secretions by gravity in the horizontal rather than the sitting posture. It is recommended that the patient be turned from side to side every hour and made to cough by the nursing and physiotherapy staff. Even with the most assiduous care, sputum retention may well occur, particularly if the patient is immobilized by traction apparatus.

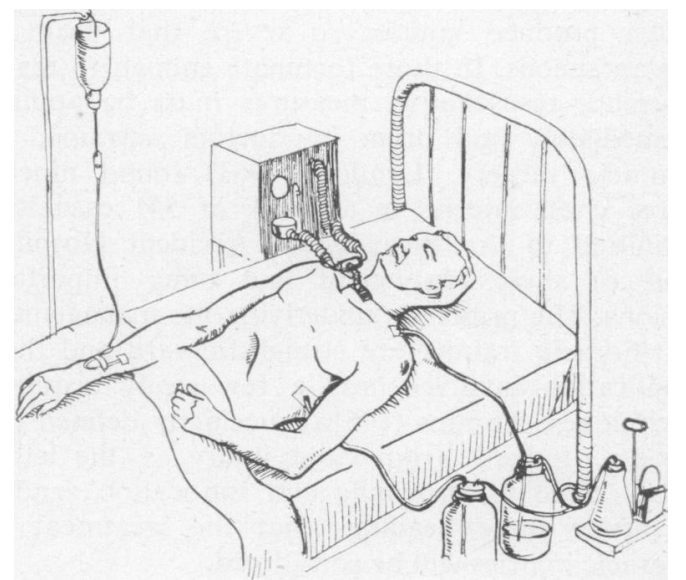

FIG. 2. Principles of management of chest injury. Patient nursed flat and rolled from side to side; mechanical ventilation via a tracheostomy; blood replacement; apical and basal intercostal catheters attached to underwater seal bottles.

Bronchoscopic aspiration of secretions may be necessary, but should sputum re-accumulate after two such aspirations, it is wise to perform a tracheostomy. Once tracheostomy has been carried out, scrupulous aseptic technique must be observed in passing suction catheters (Ambiavagar, 1967). Humidification by either instilling saline into the trachea or using a mechanical humidifier capable of saturating inspired gases with water droplets, is essential if drying of the bronchial tree with crusting of secretions and infection is to be avoided.

\section{Suffocation}

Effective respiration can only be achieved if the lung is expanded, there is a clear passage from the 
mouth to the alveoli and inflation and deflation of the lung are implemented by movement of the chest wall. Failure of any of these mechanisms can result in death from suffocation. The care of the airways has been outlined above.

\section{Maintenance of pulmonary expansion}

A pneumothorax may result from air entering the pleural cavity through the chest wall, as in a penetrating injury, from the lung itself where this has been torn by a fractured rib or from a fractured bronchus.

Pneumothorax due to penetrating injury ('sucking wound of chest') should be treated at the roadside by the application of a firm pad to the wound. In hospital the wound must be excised and sutured, and an intercostal tube inserted. Closed pneumothorax is also treated by the insertion of a tube connected to an underwater seal drain. If air bubbles out with every respiration suction should be applied to the bottle by a sucker capable of removing large volumes of air. Once full expansion of the lung has been achieved the visceral and parietal pleurae will adhere, and the air leak will seal. Rupture of the bronchus is uncommon, but should be suspected if excessive amounts of air escape and there is blood in the sputum. Sometimes rupture occurs close to the carina within the mediastinum. There may be no pneumothorax, but air tracks along the mediastinal planes into the neck and eventually the whole body becomes

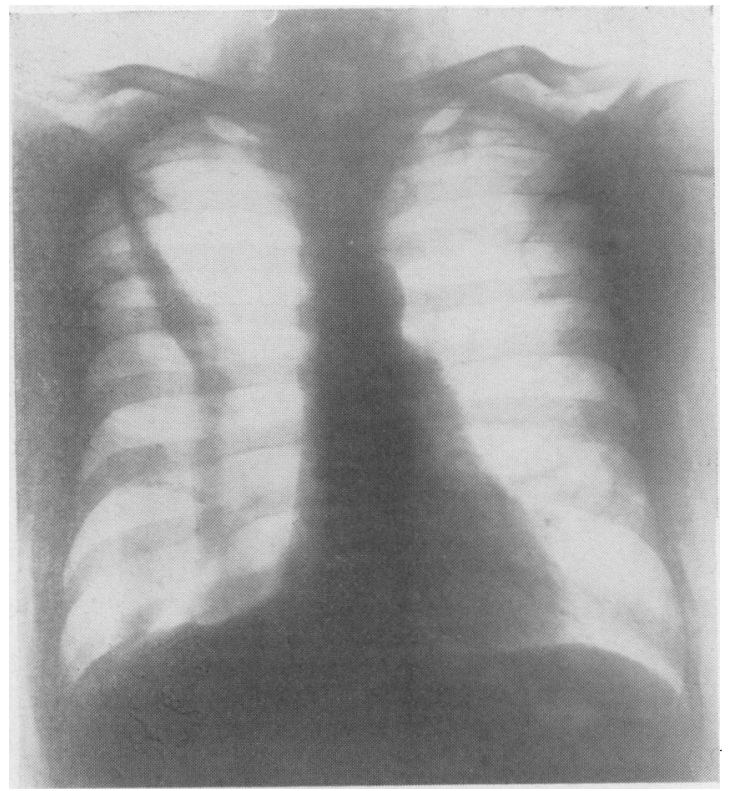

Fig. 3(a). A right-sided pneumothorax caused by a crush injury of the chest, in which there is extravasation of air into the chest wall.

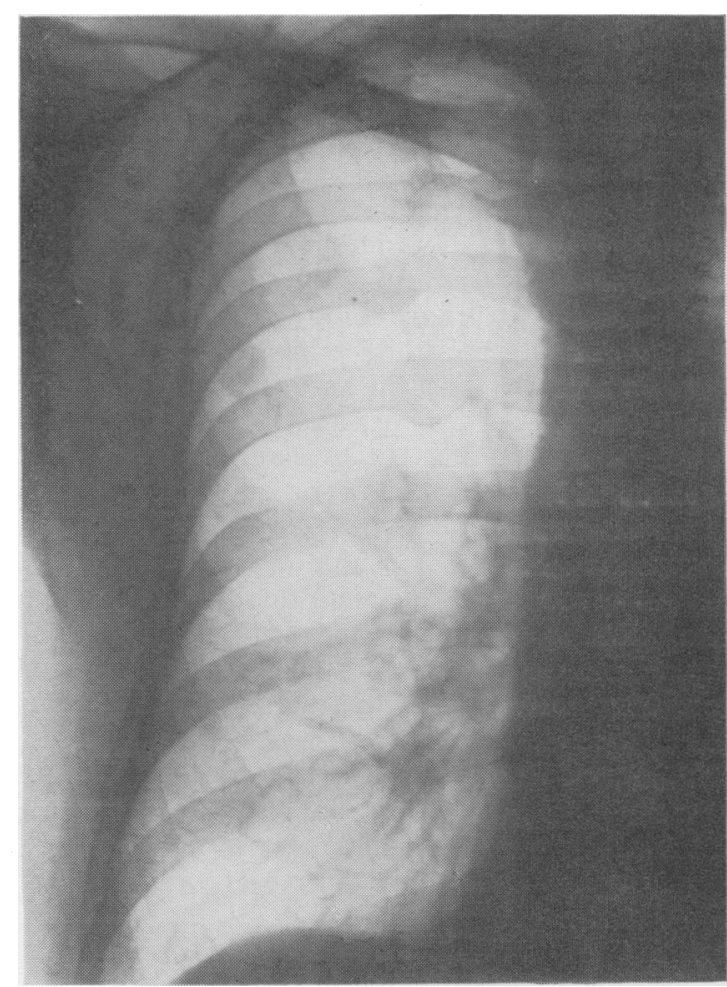

Fig. 3(b). Two weeks later, complete expansion of the lung has been achieved and callus is now apparent around the fractures of the third to eighth ribs.

bloated by surgical emphysema. Surgical emphysema may also be found when the larynx has been fractured or the cervical trachea has been avulsed from the cricoid cartilage or, commonly, when a pneumothorax escapes through a fracture in the chest wall. A crackling sensation on pressing the skin is characteristic. The presence of a tear in the bronchus is confirmed by bronchoscopy, and should be treated by immediate thoracotomy and suture of the rent (Chesterman, 1966). Compression of the lung by blood within the pleural cavity has been described above, but attention is drawn to the rare instances of rupture of the thoracic duct in crush injuries and when the thoracic spine has been forcibly extended. The escape of chyle quickly leads to a massive milky pleural effusion. This may be treated conservatively by an intercostal tube and restriction of oral fat intake, or, if the patient is well enough, by thoracotomy and ligation of the duct (Williams, 1963).

The lung may be damaged by compression injuries. Changes may range from local extravasation of blood with surrounding oedema to wide- 
spread interstitial bleeding with rupture of the alveoli. The lung assumes the consistency of liver. It is not unusual for this gross damage to occur without fracture of the ribs. Radiographic evidence of lung contusion develops from 2 to $24 \mathrm{hr}$ after injury and may then present as either a localized opacification or as widespread mottling. The patient will be dyspnoeic and cyanosed. Râles will be heard, and at bronchoscopy, blood will be seen oozing from the bronchial orifices. This blood will block the smaller bronchi and produce atelectasis. Hypoxia will be demonstrated by estimations of arterial $\mathbf{P o}_{2}$. Treatment is by intermittent positive pressure ventilation and a broad-spectrum antibiotic as there is a grave risk of super-added infection. Quite high positive pressures may be needed to secure an adequate tidal volume. If the immediate crisis can be managed, resolution of these changes can be anticipated. 'Crushed lung' is frequently associated with fractures of the pelvis (Alfano, 1965).

Rarely a lobe may be ripped from the hilum by crush injury and lie free in the pleural cavity. Such a catastrophe need not be fatal, and in fact, remarkably little bleeding may be found.

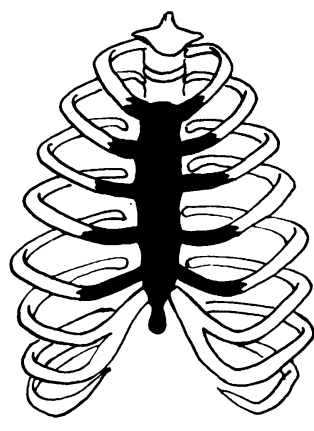

(a)

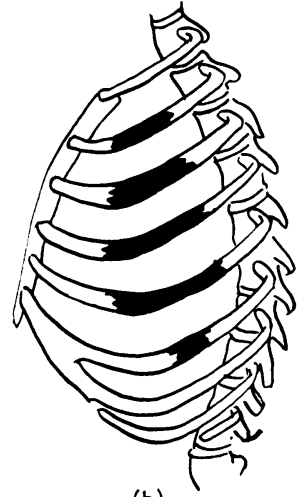

(b)
FIG. 4. Stove-in chest. (b) Lateral flail segment.

(a) Anterior flail segment.

\section{Chest wall injuries}

Compression forces applied to the rib cage or direct trauma may result in a double line of fractures. The section of chest wall between the fractures, having no attachment to the thoracic skeleton, moves independently during respiration. On inspiration, the flail segment is sucked in, and it is blown outwards during expiration. The net result is that the effect of respiratory excursion of the chest wall in ventilating the lung is neutralized. There is some evidence to show that air is not only moved up and down the trachea, but is shunted back and forth from one lung to the other. Para- doxical respiration due to a flail-segment is extremely distressing. The hypoxic patient makes desperate efforts to ventilate in the face of excruciating pain from his multiple fractures. The increased work of respiration makes further demands upon the limited oxygen available.

The first-aid worker at the roadside can do something to alleviate this distress by applying a firm pad over the loose section of chest wall. Thus, although the underlying lung is compressed, paradoxical respiration is abolished. The casualty surgeon faced with such a patient in the admitting room, who is frightened, in pain, gasping and cyanosed, can change the picture with dramatic rapidity. An intravenous relaxant drug is administered, an endotracheal tube passed and manual ventilation is commenced using either an anaesthetic machine or an Ambu bag (d'Abreu, 1964). Immediately efficient respiration is restored the patient's colour will return to normal. This simple manoeuvre should be within the compass of every casualty surgeon, and it forms the keystone in the successful management of thoracic trauma.

The definitive treatment of stove-in chest has been subject to many fluctuations and fashions. Open operation and fixation of the fractures by wiring is now usually practised if a thoracotomy has to be performed for other indications, such as persistent bleeding or rupture of the diaphragme When there is an excessive air-leak from lacerated lung, positive pressure ventilation may give rise to

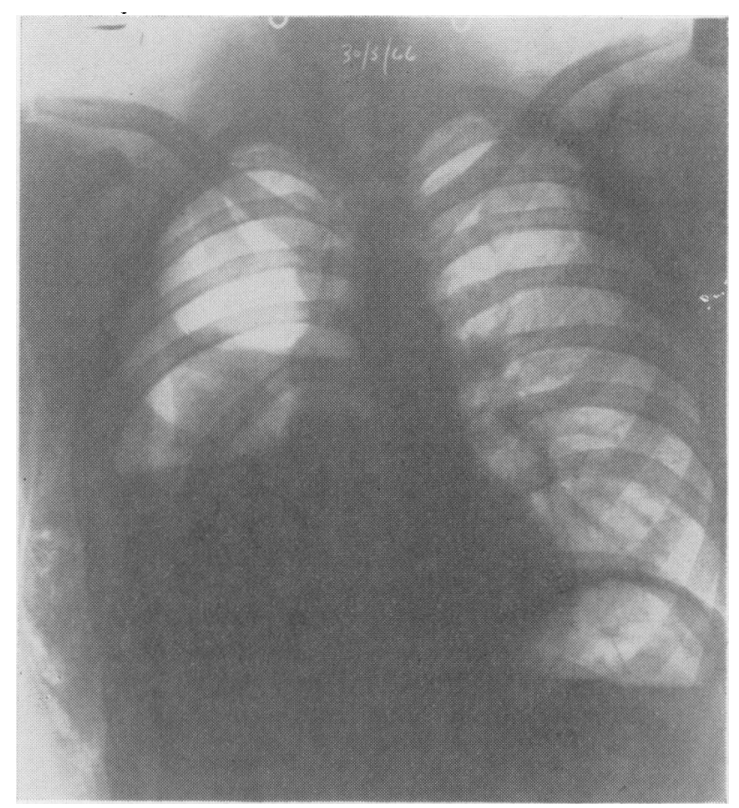

Fig. 5(a). A young man who fell $100 \mathrm{ft}$ down a cliff sustained severe chest wall injury resulting in traumatic pneumohaemothorax. 


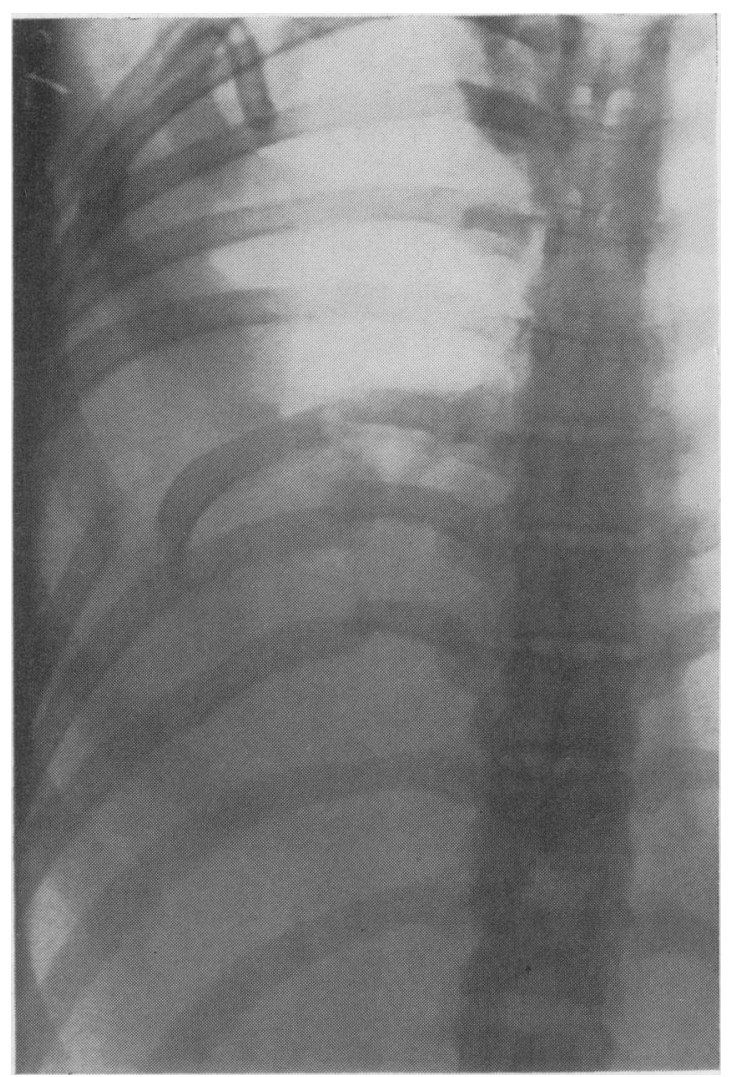

Fig. 5(b). A penetrated X-ray showing the extent of the chest wall injury. There was gross paradoxical respiration.

a tension pneumothorax despite the use of intercostal tubes. Operative fixation of the flail segment is then necessary (Scannell, 1966). Fixation may be best achieved by sutures passed through the intercostal muscles, supplemented by wires passed through holes drilled in the ribs, if the intercostals are so damaged that they will not hold stitches. The necessity for adequate blood replacement in this injury is stressed, as not only are ribs fractured, but the intercostal muscles and blood vessels are torn. When the patient has been thrown against a steering wheel, there will be a line of fractures extending down each side of the sternum or dislocation of the costochondral junctions. This anterior flail segment is best managed by passing an Abrams bar (designed for the operative treatment of pectus excavatum), behind the segment. The pointed end of the bar is inserted into the marrow cavity of a rib, the other end is lashed to a rib by wire suture. The bar is easily removed through a short incision some 3 months later (Abrams, 1961b). The practice of gripping the loose segment with towel-clips attached to traction apparatus has little to commend it. It is cumbersome and immobilizes the patient so that physiotherapy and frequent changes of posture cannot be carried out. Recently a device has been described in which hooks are passed under the mobile segment and screwed into a slotted plate, which rests on the intact chest wall. The writer has no experience of this interesting development.

The basis of current management of flail chest is 'internal pneumatic fixation'. The lungs are inflated by intermittent positive pressure applied by a mechanical ventilator so that the loose segment is held outward on a cushion of expanded air. As at least 10 days are necessary before any degree of consolidation of the segment can be expected it will be necessary to perform a tracheostomy. There is no urgency to do this, and initial management with an indwelling endotracheal tube is acceptable.

Transverse fractures of the sternum have been described after excessive muscular activity, and even during labour. They may also be associated with acute flexion injuries in which crush fractures of the thoracic vertebrae are sustained. Rarely, one section of the sternum may be driven backwards and ledge under the other, thereby compressing mediastinal structures. Open reduction and fixation may be necessary. In those instances where a stove-in chest is associated with a frac-

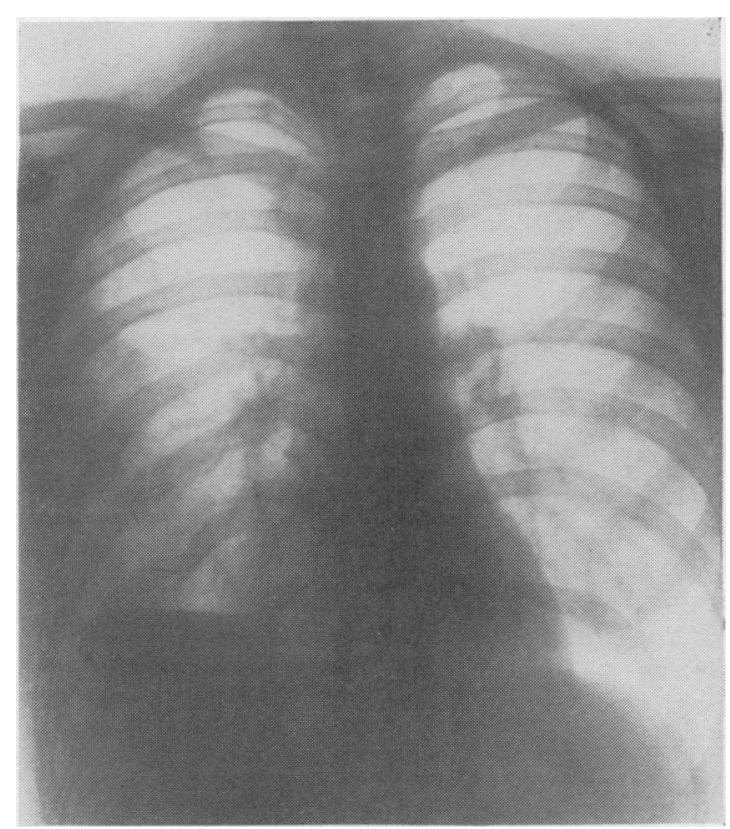

FIG. 5(c). The same patient after evacuation of the clotted haemothorax at thoracotomy with wiring of the fractured ribs. 
tured clavicle, the pull of the shoulder girdle muscles draws the humerus medially and the flail segment is pushed inwards. The end result of this injury, if left untreated, is a chest deformity similar to that produced by a thoracoplasty. Open reduction and fixation of the clavicle is essential.

Patients being treated with a mechanical ventilator should have regular estimations of $\mathrm{Po}_{2}$, $\mathrm{PCO}_{2}$ and serum bicarbonate, to ensure that adequate respiration is being achieved. Unrelieved respiratory acidosis and hypoxia will be deleterious in head injuries and will impair cardiac function. The fall in cardiac output thus produced will lead to metabolic acidosis and perhaps renal failure. The interplay of these factors has been well described by Reid \& Baird (1965). This method of treating stove-in chest was introduced by Avery, Morch \& Benson (1965).

Respiratory

dysfunction
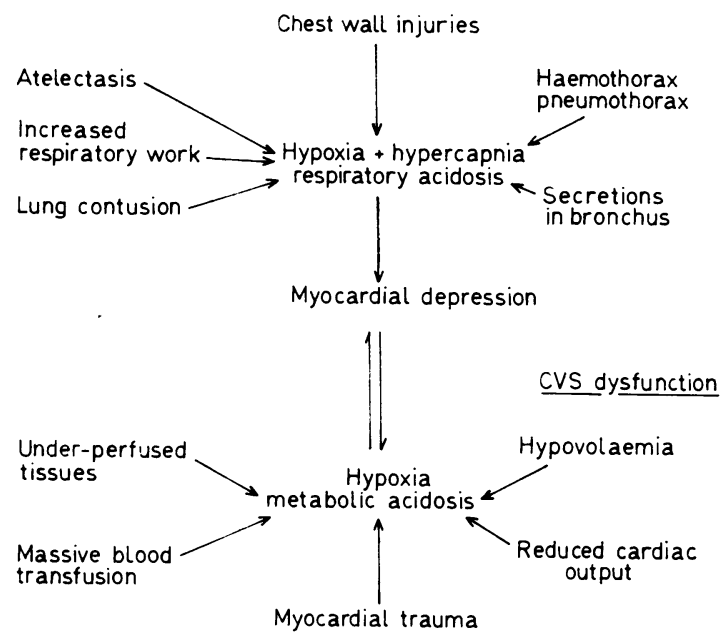

Fig. 6. Factors producing metabolic and respiratory acidosis in chest injuries. Modified from Reid \& Baird (1965).

\section{Rupture of the diaphragm}

It is easy to forget that the diaphragm constitutes an important part of the chest wall. If it is torn or paralysed respiration can be seriously embarrassed.

Crush injuries of the lower chest and upper abdomen frequently result in rupture of the diaphragm. Such tears may be either radial or circumferential. The colon and stomach on the left, and the liver on the right, are thrust up into the thorax, and the lung is compressed. The patient may complain of shoulder-tip pain, and bowel sounds are sometimes heard on auscultation of the chest. The diagnosis is confirmed on radiography

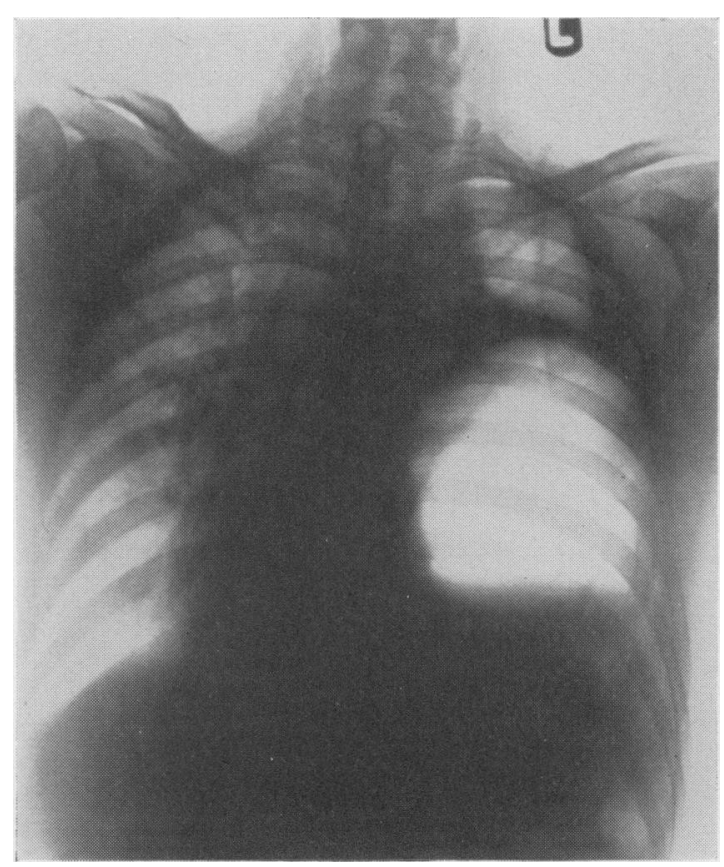

FIG. 7. Traumatic rupture of the left dome of the diaphragm with herniation of the stomach into the left pleural cavity.

when the bowel will be seen above the level of the diaphragm. Early surgery is necessary. The chest is explored through a low (eighth rib) thoracotomy. The two halves of the diaphragm tend to roll up so that at first sight there seems to be an alarming deficiency in tissue. Once the diaphragm has been unrolled it is almost always possible to carry out a repair by direct suture, and only rarely will a prosthetic patch be needed. Concomitant injury to the spleen or liver can easily be dealt with through the chest. However, it is as well to remember the limitations of a thoracic approach. Tears of the mesentery and rupture of the duodenum may well be missed if the abdomen is explored through the diaphragm. If there is evidence of peritonitis or intraperitoneal bleeding it is wisest to use a standard laparotomy incision and repair the diaphragm from below. An associated pneumothorax or haemopneumothorax must be treated first by the insertion of an intercostal tube, otherwise the anaesthetist will produce a tension pneumothorax when he inflates the lungs.

\section{Injuries of the heart and great vessels}

Injuries of the heart

The pattern of violence in Britain is fortunately not such that the experience of Beall et al. (1961) and Boyd \& Streider (1965) in the management 
of stab wounds of the heart can be matched but the casualty officer will occasionally be faced with a patient who has survived a penetrating cardiac injury long enough to reach hospital. Where the knife-thrust has created a sufficiently large hole in the pericardium, blood will escape into the pleural cavity and the problem is that of immediate blood replacement and urgent surgery to suture the wound in the heart. Where, as is usually the case, the pericardial wound is small, blood collects within the pericardial sac and compresses the heart. Both atrial and ventricular filling are impaired. The former is manifested by distension of the neck veins and hepatic enlargement, the latter by a low output state with a low systemic pressure and the signs of shock. Pulsus paradoxus, in which the normal slight fall in systolic pressure on inspiration is exaggerated, may be detected.

The heart sounds are muffled, cardiac enlargement may be seen on the chest radiograph and there may be low voltage QRS complexes on the electrocardiogram. The signs of raised venous pressure may well be missing in the presence of a low circulating blood volume. Immediate improvement is produced by aspirating the pericardium. A long needle is inserted to the left of the xiphisternum and directed upwards and slightly backwards. The sensation of passing through the pericardium is not often elicited and confirmation that the needle has been inserted to the correct depth is obtained, somewhat alarmingly, by feeling the ventricle scrape on the tip of the needle. If a unipolar electrocardiograph lead is connected to the needle, an injury pattern will be recorded when the heart is touched. A single aspiration may be all the treatment necessary as cardiac wounds seal rapidly, but should the signs of cardiac tamponade recur, immediate operation must be carried out. The heart is exposed through a left anterior thoracotomy, and the laceration is closed with interrupted silk sutures. Bleeding is controlled by compressing the lips of the incision between finger and thumb.

The heart may be severely contused in compression injuries and treatment with digitalis and diuretics may be needed for heart failure. Abnormal rhythms can develop and the electrocardiograph will reveal an infarction pattern with elevation of the SGOT levels. Bed rest for 3 or 4 weeks is advisable after such an injury. Defects in both the atrial and ventricular septa have been described following crush and penetrating injuries. Successful closure of these defects on cardiopulmonary by-pass has been achieved as has repair of ruptured papillary muscles, chordae tendineae and cardiac valves (Dunseth \& Ferguson, 1965).

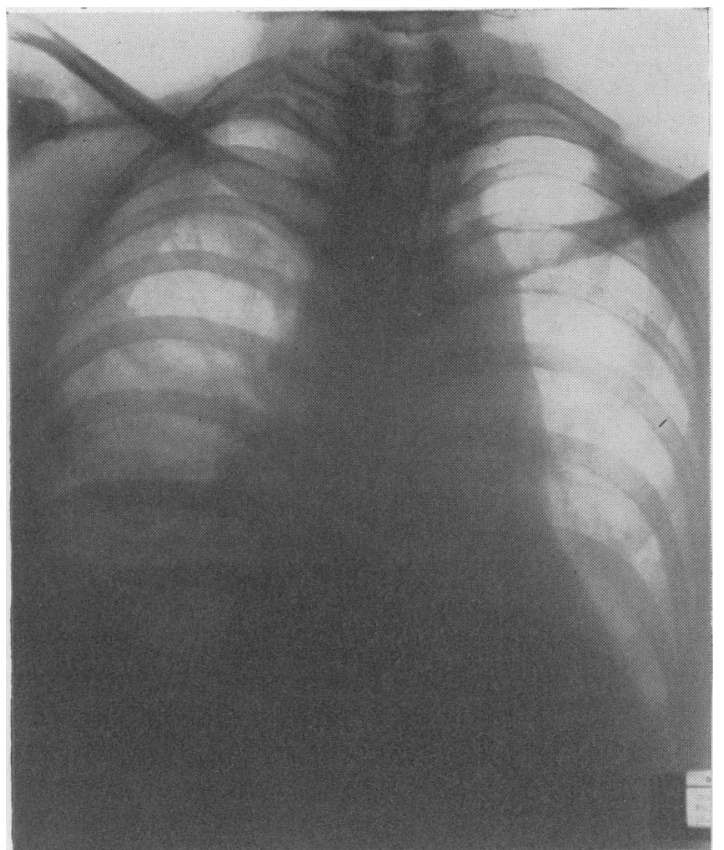

FIG. 8. Traumatic rupture of the aorta. Note the broad mediastinum and the fractures of the first and second ribs.

\section{Traumatic rupture of the aorta}

In head-on collisions, the great decelerating forces engendered apply a shearing stress between the arch and descending thoracic aorta. Pressures of many hundreds of millimetres of mercury are developed within the vessel and rupture characteristically occurs just distal to the left subclavian artery at that point where the ligamentum arteriosum is attached. In many cases death is instantaneous, but sometimes the adventitia remains intact for hours, weeks, and, rarely, for years. The first prerequisite for making the diagnosis is a high index of suspicion in all patients who have been involved in a collision. Sometimes only minor violence may be sustained. The superior mediastinum will be seen to be broadened on the chest radiograph, and there may be a bloody pleural effusion, sometimes with hoarseness and dysphagia. An aortogram will be conclusive in doubtful cases. Immediate surgery must be undertaken if these patients are to be saved as the adventitia may give way at any time. The aorta is exposed through a left thoracotomy and left atrial-to-femoral artery by-pass is commenced. This simple form of extracorporeal circulation consists essentially of a length of PVC tubing and a roller pump. Blood is drawn from the left atrium and returned to the femoral artery so that when 
the aorta is clamped the blood pressure in the head is kept at normal levels and the lower half of the body is perfused.

The damaged segment of the aorta is excised and replaced by a prosthetic graft. It is seldom possible to perform a direct anastomosis (McClenathen \& Brettschneider, 1965). Less commonly, the innominate artery is avulsed from the aortic arch by the crushing injury.

\section{Summary and conclusions}

Perhaps the greatest problem for those dealing with patients who have sustained multiple injuries is the decision of priorities. Trauma to the head, the trunk and the limbs demands urgent resuscitative measures, but where is the surgeon to start? The injured chest should surely demand prior attention as the combination of blood-loss and hypoxia can be so rapidly lethal. It is suggested that when the patient is admitted a rapid clinical evaluation of the injuries is made, blood is taken for cross-matching and an intravenous infusion of blood or plasma-expanding solution is commenced.

If the patient is cyanosed and distressed with an obvious paradoxical segment of chest wall an intravenous relaxant drug is given, an endotracheal tube is passed and the patient is manually inflated with oxygen.

Now that the immediate threat to life has been removed, a more thorough examination can be made, and radiographs may be taken. To delay these measures while the patient is taken to the $\mathrm{X}$-ray department is to court disaster. If the films confirm the presence of a pneumothorax or haemothorax, an intercostal tube is inserted. During the next few hours thoracotomy may be necessary for bleeding into the pleural or pericardial cavities, or for rupture of the aorta, bronchus or diaphragm. Exploration of the abdomen or skull and the care of compound limb fractures may also be required. The decision to perform a tracheostomy may be safely deferred until 2 or 3 days later. At all times the possibility of obstruction of the respiratory passages by blood or sputum must be kept in mind. If an indwelling endotracheal tube or tracheostomy is available, patency of the airways can be maintained by assiduous nursing care. If not, then urgent bronchoscopic aspiration of secretions must be performed. The deleterious effect of hypoxia upon an associated brain injury is well known. The successful management of thoracic trauma calls upon all the resources of medical and nursing staff. The advantages of a fully equipped intensive care unit, which is brightly illuminated and fully staffed, both night and day, cannot be too greatly stressed. Careful observation and the prompt application of the relatively few therapeutic measures outlined above form the basis of the successful treatment of thoracic trauma.

\section{Acknowledgments}

I am grateful to Professor A. L. d'Abreu and Mr L. D. Abrams for their helpful criticism and to $\mathrm{Mr} \mathrm{T}$. Dee for reproducing the radiographs.

\section{References}

Abrams, L.D. (1961a) Non-penetrating injuries of the chest and abdomen. Proc. roy. Soc. Med. 54, 561.

ABraMs, L. D. (1961b) Operative treatment of Funnel Chest. Acta chir. belg. 2, 11.

Alfano, G.S. (1965) Pulmonary contusion. J. Trauma, 5, 647.

Ambiavagar, M. (1967) Treatment of respiratory failure following chest injuries. Postgrad. med. J. 43, 256.

Avery, E.E., Morch, E.T. \& BENSON, D.W. (1956) Criticallyô crushed chests. J. thorac. Surg. 32, 291.

Beall, A.C., Crawford, H. \& DeBaKey, M.E. (1966) Considerations in the management of acute traumatic haemothorax. J. thorac. Surg. 52, 351.

Beall, A.C., Oschner, J.L., Morris, G.C., Cooley, D.A. \& DeBakey, M.E. (1961) Penetrating wounds of the heart. J. Trauma, 1, 195.

BoYD, T.F. \& Streider, J.W. (1965) Immediate surgery for traumatic heart disease. J. thorac. Surg. 50, 305.

Chesterman. J.T. (1966) Rupture of the trachea and bronchi. Thorax, 21, 21.

D'Abreu, A.L. (1964) Thoracic injuries. J. Bone Jt. Surg. 46(B), 581 .

Dunseth, W. \& Ferguson, T. (1965) Acquired cardiac septal defect due to thoracic trauma. J. Trauma, 5, 142.

London, P.S. (1963) Multiple injuries. Proc. roy. Soc. Med. 56, 821.

McClenathan, J.E. \& Brettschneider, L. (1965) Traumatic thoracic aortic aneurysms. J. thorac. Surg. 50, 74.

Price-Thomas, C. \& Cleland, W.P. (1945) Decortication in clotted and infected haemothoraces. Lancet, i, 327.

REID, J.M. \& BAIRD, W.L.M. (1965) Crushed chest injuries, some physiological disturbances and their correction. Brit. med. J. i, 1105.

SCANNELl, J.G. (1966) Surgical management of major chest injuries. Surg. Clin. N. Amer. 46, 539.

Williams, K.R. (1963) The management of chylothorax related to trauma. J. Trauma, 3, 317 . 